\title{
Genome Sequence of Fusarium oxysporum f. sp. conglutinans, the Etiological Agent of Cabbage Fusarium Wilt
}

\author{
Fangwei Yu, ${ }^{1}$ Wei Zhang, ${ }^{1}$ Shenyun Wang, ${ }^{1}$ Hong Wang, ${ }^{1}$ Li Yu, ${ }^{1}$ Xiaoping Zeng, ${ }^{2}$ Zhangjun Fei, ${ }^{3, \dagger}$ \\ and Jianbin $\mathrm{Li}^{1, \dagger}$ \\ 1 Jiangsu Key Laboratory for Horticultural Crop Genetic Improvement, Institute of Vegetable Crops, \\ Jiangsu Academy of Agricultural Sciences, Nanjing 210014, China \\ 2 Jiangsu Agricultural Technology Extension Station, Nanjing 210036, China \\ ${ }^{3}$ Boyce Thompson Institute, Cornell University, Ithaca, NY 14853, U.S.A.
}

\begin{abstract}
Fusarium oxysporum f. sp. conglutinans is the causal agent of Fusarium wilt of cabbage (Brassica oleracea var. capitata L.), which results in severe yield loss. Here, we report a highquality genome sequence of a race 1 strain (IVC-1) of $F$. oxysporum $f$. sp. conglutinans, which was assembled using a combination of PacBio long-read and Illumina short-read sequences. The assembled IVC-1 genome has a total size of $71.18 \mathrm{Mb}$, with a contig N50 length of 4.59 $\mathrm{Mb}$, and encodes 23,374 predicted protein-coding genes. The high-quality genome of IVC-1 provides a valuable resource for facilitating our understanding of $F$. oxysporum f. $\mathrm{sp}$. conglutinans-cabbage interaction.
\end{abstract}

\section{Genome Announcement}

Fusarium oxysporum is a ubiquitous soil-inhabiting fungus that infects more than 100 different plant species worldwide (Ma et al. 2013; Michielse and Rep 2009). F. oxysporum is also notorious for causing opportunistic infections in immunocompromised patients (Nucci and Anaissie 2007). Due to its great scientific and economic importance, $F$. oxysporum has been nominated as one of the top 10 fungal pathogens (Dean et al. 2012). The infection of plants by pathogenic $F$. oxysporum involves several steps, including root recognition, root surface attachment and colonization, penetration and colonization of the root cortex, and hyphal growth within the xylem vessels (Di Pietro et al. 2003). The key to successful invasion by $F$. oxysporum depends on its ability to properly recognize the host roots by sensing secreted plant peroxidases through the mitogen-activated protein kinase cascade and the seven-pass transmembrane protein Ste2 (Turrà et al. 2015). Differing in host specificity, F. oxysporum can be classified into more than 120 formae speciales, some of which can be further divided into several physiological races (Edel-Hermann and Lecomte 2019; Gordon and Martyn 1997; Michielse and Rep 2009).

F. oxysporum f. sp. conglutinans is the causal agent of cabbage Fusarium wilt (CFW), which poses a great threat to global cabbage production. F. oxysporum f. $\mathrm{sp}$. conglutinans populations with high pathogenicity have been emerging in China in recent years (Liu et al. 2017). To date, only two races have been documented in $F$. oxysporum f. sp. conglutinans (Morrison et al. 1994; Ramirez-Villupadua et al. 1985), and race 1 is the dominant strain in

${ }^{\dagger}$ Corresponding authors: J. Li; jbli@jaas.ac.cn and Z. Fei; zf25@cornell.edu

The author(s) declare no conflict of interest.

Accepted for publication 15 October 2020.

\section{Funding}

This work was supported by grants from the China Agriculture Research System (CARS-25), and the Natural Science Foundation of Jiangsu Province (BK20170608).

\section{Keywords}

Brassica oleracea var. capitata L., Fusarium oxysporum f. sp. conglutinans, genome, microbe-plant interaction 

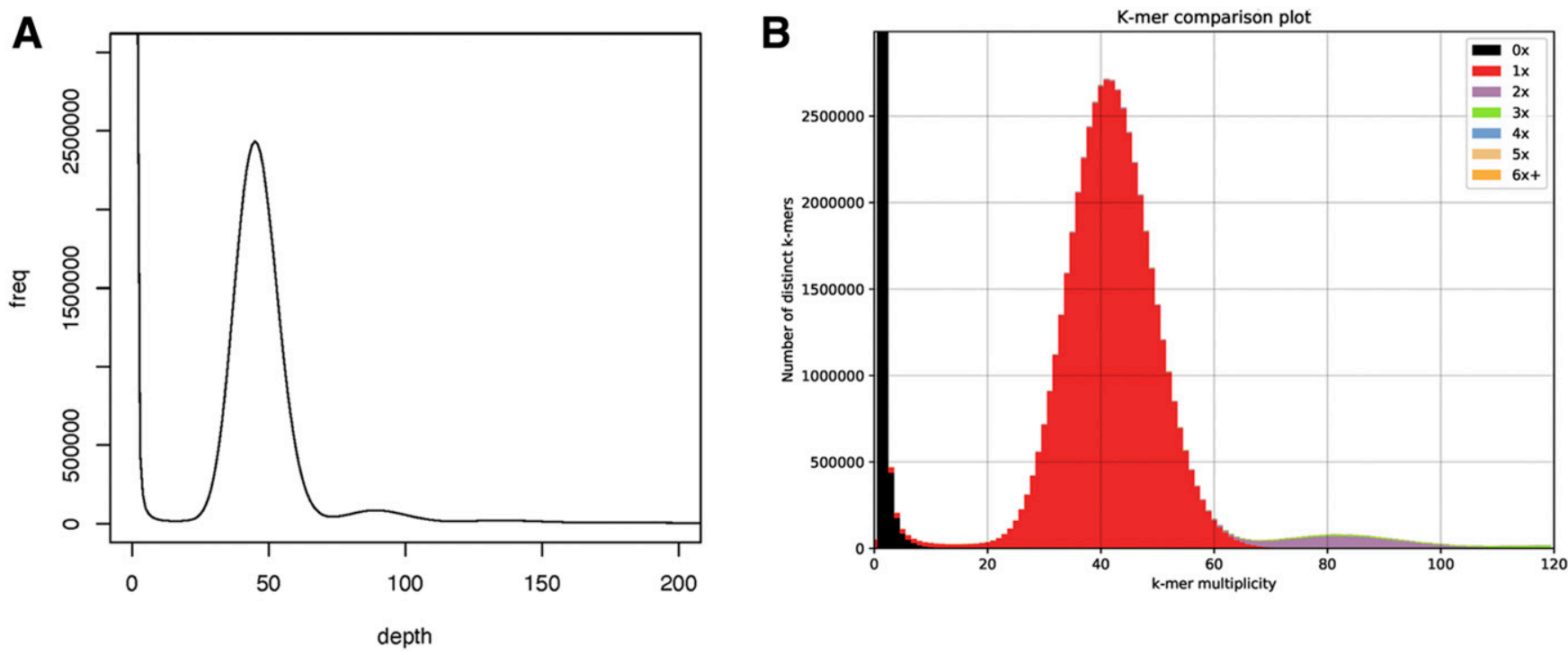

Fig. 1. K-mer analysis of Fusarium oxysporumf. sp. conglutinans genome. A, The 17-mer depth distribution of the lllumina paired-end reads. A total of 3,325,251,032 distinct 17-mers were recovered, with a peak depth of 45 . The genome size was estimated based on the following formula: genome size $=($ total number of $\mathrm{k}$-mers $) /($ position of peak depth $)=3,325,251,032 / 45=73.89 \mathrm{Mb}$. B, Comparison of 17 -mer spectra between the Illumina paired-end reads and the genome assembly.

Table 1. Genome statistics of Fusarium oxysporum f. sp. conglutinans race 1 (IVC-1 and FGL03-6), race 2 (54008), and F. oxysporum f. sp. lycopersici 4287 (Fol4287)

\begin{tabular}{lcccc} 
Strain & IVC-1 (race 1) & FGL03-6 (race 1) & 54008 (race 2) & Fol4287 \\
Genome size $(\mathrm{Mb})$ & 71.18 & 61.66 & 53.58 & 61.39 \\
Number of scaffolds & 55 & 414 & 2,552 & 0.16 \\
Scaffold N50 $(\mathrm{Mb})$ & 5.00 & 1.21 & 3,350 & 1.98 \\
Number of contigs & 64 & 1,079 & 0.09 & 1,362 \\
Contig N50 (Mb) & 4.59 & 0.34 & 26,246 & 0.10 \\
Number of genes & 23,374 & 9,790 & $47.70 \%$ & 27,347 \\
GC content & $48.19 \%$ & $48.00 \%$ & $99.2 \%$ & $96.43 \%$ \\
BUSCO completeness & $99.4 \%$ & $99.2 \%$ & $96.4 \%$ \\
\hline
\end{tabular}

China (Liu et al. 2019). Improving our current knowledge of the molecular mechanisms underlying pathogenesis and host specificity of $F$. oxysporum $\mathrm{f}$. $\mathrm{sp}$. conglutinans races is fundamental for CFW management. To this end, it would be very valuable to obtain highquality genome sequences for $F$. oxysporum $\mathrm{f}$. sp. conglutinans races.

Genome assemblies have been released for $F$. oxysporum f. sp. conglutinans race 1 FGL03-6 (NRIA00000000.2) and race 254008 (AGNF00000000.1). However, both genomes were assembled using Illumina short reads and were thus highly fragmented, making it challenging to perform in-depth analyses of genomic features that associate with variations and virulence among F. oxysporumf. sp. conglutinans races. Here, we report the high-quality genome sequence of a race 1 strain of $F$. oxysporum f. sp. conglutinans, IVC-1, which was initially isolated from roots of $F$. oxysporum f. sp. conglutinans-infected cabbage collected in North China. Genomic DNA was extracted from the mycelia of IVC-1 and was used to construct an Illumina paired-end library with an insert size of approximately $270 \mathrm{bp}$, using the TruSeq Nano DNA high throughput library prep kit (Illumina) and a PacBio single-molecule real-time (SMRT) library, with an insert size of approximately $20 \mathrm{~kb}$, using the SMRTbell template prep kit 1.0 (PacBio), following manufacturer instructions. The Illumina paired-end library was sequenced on an Illumina HiSeq X Ten platform, yielding a total of $3.7 \mathrm{~Gb}$ of highquality sequences, with a read length of $150 \mathrm{bp}$, which represented approximately 52x coverage of the IVC-1 genome that had an estimated size of $73.89 \mathrm{Mb}$, based on the k-mer depth distribution analysis of the Illumina short reads (Fig. 1A). The PacBio SMRT library was sequenced on four SMRT cells of a PacBio Sequel system, which yielded a total of $18.9 \mathrm{~Gb}$ 
(approximately $265 \times$ coverage of the IVC-1 genome) long-read data with read N50 and mean lengths of 22,160 and $16,911 \mathrm{bp}$, respectively.

The PacBio long reads were de novo assembled using Falcon (v0.3.0) (Chin et al. 2016), with parameters '- $v$-dal8 -t32 -h60 -e.96 -1500 -s100 -H3000'. The resulting assembled contigs were corrected for base errors using the Illumina reads with GATK software (available online). The corrected contigs were further connected into scaffolds, using SSPACE (v2.0) (Boetzer et al. 2011) with the Illumina reads, and gap filling of scaffolds was then performed, using PBJelly2 (v15.8.24) (English et al. 2012). The final assembled genome of IVC-1 had a total length of $71,184,270 \mathrm{bp}$ and a GC content of $48.19 \%$ (Table 1). The assembly comprised 64 contigs with an $\mathrm{N} 50$ length of $4.59 \mathrm{Mb}$ and 55 scaffolds with an N50 length of $5.00 \mathrm{Mb}$. The assembled genome of IVC-1 (71.18 Mb) was much larger than those of $F$. oxysporum $\mathrm{f} . \mathrm{sp}$. conglutinans race 1 FGL03-6 (NRIA00000000.2, $61.66 \mathrm{Mb}$ ), F. oxysporum f. sp. conglutinans race 254008 (AGNF00000000.1, $53.58 \mathrm{Mb}$ ), and F. oxysporum f. sp. lycopersici 4287 (AAXH00000000.1, 61.39 Mb) and also had much better continuity than the other three F. oxysporum assemblies, indicated by a 13- to 50-fold improvement of contig N50 length in IVC-1 assembly compared with other three (Table 1). The completeness of the IVC-1 assembly was assessed using BUSCO (v3.0.2) (Simão et al. 2015). Of the 1,315 single-copy orthologs in the ascomycota data set, 1,306 (99.4\%) were found complete in the IVC-1 assembly (Table 1).

Comparison of k-mers in Illumina paired-end reads and those in the IVC-1 assembly revealed that almost the entire assembly was covered by single-copy 17-mers (1x), while a very small portion of $k$-mers with $\geq 2 \times$ coverage represented the repetitive sequences in the assembly (Fig. 1B), confirming that there were no redundant sequences in the assembly. Blast analysis of the assembly against the GenBank nucleotide database showed that all hits were from fungi, with $99 \%$ of the hits from Fusarium, suggesting there was no contamination in the assembly. Alignment of the assembly to other Fusarium genomes showed that the aligned scaffolds exhibited high collinearity with published Fusarium genomes, while, as expected, some scaffolds failed to align. In summary, the size of this genome assembly is larger than other published Fusarium genomes, but there is no evidence of contamination or redundancy in this assembly, and the assembled genome size of IVC-1 (71.18 Mb) is close to the estimated genome size (73.89 Mb). Fungal genomes evolve very fast, especially on the dispensable chromosomes (Peng et al. 2019; Sperschneider et al. 2015), which are normally divergent between species or even strains. In addition, this genome was assembled using the long-read technology and, therefore, could include some regions that were not assembled in other assemblies with short-read sequences.

A total of 23,374 protein-coding genes were predicted in the genome of IVC-1 by integrating homology predictions, using GeneWise (v2.20) (Birney et al. 2004), and de novo predictions using SNAP (v2010-07-28) (Korf 2004), Augustus (v3.2.1) (Stanke et al. 2008), and GeneMark-ES (v4.21) (Ter-Hovhannisyan et al. 2008). The number of predicted genes in IVC-1 was comparable to those in 54008 and Fol4287 but more than that in FGL03-6 (Table 1).

The high-quality genome assembly of the race 1 strain of $F$. oxysporum f. sp. conglutinans, IVC-1, provides a valuable resource that will enable in-depth analysis of Fusarium pathogenesis and interaction between $F$. oxysporum f. sp. conglutinans and cabbage and will facilitate the development of better control approaches against CFW.

This whole-genome shotgun project has been deposited at DDBJ/ENA/GenBank under the accession JABTBS000000000. Raw PacBio and Illumina sequencing reads have been deposited into National Center for Biotechnology Information Short Read Archive under the accessions SRR11823423 and SRR11823424,respectively.

\section{Author-Recommended Internet Resource}

The Genome Analysis Toolkit (GATK): https://www.broadinstitute.org/gatk

\section{Literature Cited}

Birney, E., Clamp, M., and Durbin, R. 2004. GeneWise and Genomewise. Genome Res. 14:988-995.

Boetzer, M., Henkel, C. V., Jansen, H. J., Butler, D., and Pirovano, W. 2011. Scaffolding pre-assembled contigs using SSPACE. Bioinformatics 27:578-579.
Chin, C. S., Peluso, P., Sedlazeck, F. J., Nattestad, M., Concepcion, G. T., Clum, A. Dunn, C., O'Malley, R., Figueroa-Balderas, R., Morales-Cruz, A., Cramer, G. R., Delledonne, M., Luo, C., Ecker, J. R., Cantu, D., Rank, D. R., and Schatz, M. C. 2016. Phased diploid genome assembly with single-molecule real-time sequencing. Nat. Methods 13:1050-1054. 
Dean, R., Van Kan, J. A. L., Pretorius, Z. A., Hammond-Kosack, K. E., Di Pietro, A., Spanu, P. D., Rudd, J. J., Dickman, M., Kahmann, R., Ellis, J., and Foster, G. D. 2012. The Top 10 fungal pathogens in molecular plant pathology. Mol. Plant Pathol. 13:414-430.

Di Pietro, A., Madrid, M. P., Caracuel, Z., Delgado-Jarana, J., and Roncero, M. I. 2003. Fusarium oxysporum: Exploring the molecular arsenal of a vascular wilt fungus. Mol. Plant Pathol. 4:315-325.

Edel-Hermann, V., and Lecomte, C. 2019. Current status of Fusarium oxysporum formae speciales and races. Phytopathology 109:512-530.

English, A. C., Richards, S., Han, Y., Wang, M., Vee, V., Qu, J., Qin, X., Muzny, D. M., Reid, J. G., Worley, K. C., and Gibbs, R. A. 2012. Mind the gap: Upgrading genomes with Pacific Biosciences RS long-read sequencing technology. PLoS One 7:e47768.

Gordon, T. R., and Martyn, R. D. 1997. The evolutionary biology of Fusarium oxysporum. Annu. Rev. Phytopathol. 35:111-128.

Korf, I. 2004. Gene finding in novel genomes. BMC Bioinformatics 5:59.

Liu, X., Ling, J., Xiao, Z., Xie, B., Fang, Z., Yang, L., Zhang, Y., Lv, H., and Yang, Y. 2017. Characterization of emerging populations of Fusarium oxysporum f. sp. conglutinans causing cabbage wilt in China. J. Phytopathol. 165:813-821.

Liu, X., Xing, M., Kong, C., Fang, Z., Yang, L., Zhang, Y., Wang, Y., Ling, J., Yang, Y., and Lv, H. 2019. Genetic diversity, virulence, race profiling, and comparative genomic analysis of the Fusarium oxysporum $\mathrm{f}$. $\mathrm{sp}$. conglutinans strains infecting cabbages in China. Front. Microbiol. 10:1373.

Ma, L. J., Geiser, D. M., Proctor, R. H., Rooney, A. P., O'Donnell, K., Trail, F., Gardiner, D. M., Manners, J. M., and Kazan, K. 2013. Fusarium pathogenomics. Annu. Rev. Microbiol. 67:399-416.

Michielse, C. B., and Rep, M. 2009. Pathogen profile update: Fusarium oxysporum. Mol. Plant Pathol. 10:311-324.
Morrison, R. H., Mengistu, A., and Williams, P. H. 1994. First report of race 2 of cabbage yellows caused by Fusarium oxysporumf. sp. conglutinans in Texas. Plant Dis. 78:641.

Nucci, M., and Anaissie, E. 2007. Fusarium infections in immunocompromised patients. Clin. Microbiol. Rev. 20:695-704.

Peng, Z., Oliveira-Garcia, E., Lin, G., Hu, Y., Dalby, M., Migeon, P., Tang, H., Farman, M., Cook, D., White, F. F., Valent, B., and Liu, S. 2019. Effector gene reshuffling involves dispensable mini-chromosomes in the wheat blast fungus. PLoS Genet. 15:e1008272.

Ramirez-Villupadua, J., Endo, R. M., Bosland, P., and Williams, P. H. 1985. A new race of Fusarium oxysporum $f$. sp. conglutinans that attacks cabbage with type $A$ resistance. Plant Dis. 69:612-613.

Simão, F. A., Waterhouse, R. M., loannidis, P., Kriventseva, E. V., and Zdobnov, E. M. 2015. BUSCO: Assessing genome assembly and annotation completeness with single-copy orthologs. Bioinformatics 31:3210-3212.

Sperschneider, J., Gardiner, D. M., Thatcher, L. F., Lyons, R., Singh, K. B., Manners, J. M., and Taylor, J. M. 2015. Genome-wide analysis in three Fusarium pathogens identifies rapidly evolving chromosomes and genes associated with pathogenicity. Genome Biol. Evol. 7:1613-1627.

Stanke, M., Diekhans, M., Baertsch, R., and Haussler, D. 2008. Using native and syntenically mapped cDNA alignments to improve de novo gene finding. Bioinformatics 24:637-644.

Ter-Hovhannisyan, V., Lomsadze, A., Chernoff, Y. O., and Borodovsky, M. 2008. Gene prediction in novel fungal genomes using an ab initio algorithm with unsupervised training. Genome Res. 18:1979-1990.

Turrà, D., El Ghalid, M., Rossi, F., and Di Pietro, A. 2015. Fungal pathogen uses sex pheromone receptor for chemotropic sensing of host plant signals. Nature 527: 521-524. 